\title{
Comportamiento hídrico de pizarras de techar
}

RUIZ DE ARGANDOÑa, V. G. y CALLEJA, L Grupo de Petrofísica. Dpto. de Geologia. Universidad de Oviedo

\begin{abstract}
RESUMEN
Se estudia el comportamiento hídrico de dos rocas pizarrosas del NW de España (filita de Rande - La Coruña-y pizarra de Luarca -Asturias-), utilizadas como pizarras ornamentales y de techar. Se propone una metodología experimental para una correcta interpretación de los fenómenos hidricos a que pueden estar sometidas por sus usos en la industria de la construcción. Los resultados de los ensayos (hinchamiento, desmoronamiento y ciclos humedad/sequedad) se relacionan con la mineralogía y textura, asi como con algunas propiedades físicas relacionadas con el movimiento del agua por el interior de las rocas (porosidad, permeabilidad al aire y absorción de agua).
\end{abstract}

\section{SUMMARY}

The hidric behaviour of two slaty rocks from the NW of Spain (phyllite of Rande - La Coruña- and slate of Luarca -Asturias-) used as ornamental and roof slate is studied. An experimental methodology for proper interpretation of the hidric phenomena to which they can be subjected due to their particular use in the building industries is proposed. The results of the tests (swelling, slaking and wetting/drying cycles) are related to the mineralogy and texture, as well as to some physical properties related to the water movement throughout the rocks (porosity, air permeability and water absorption).
PALABRAS Clave:

ROCA INDUSTRIAL, PIZARRAS, PROPIEDADES HIDRICAS, PETROFÍSICA.

\section{INTRODUCCIÓN}

Es un hecho extendido que un gran número de rocas, principalmente pizarrosas, sufren cambios apreciables cuando se exponen a cambios en las condiciones de humedad, o bajo la acción directa del agua. La sensibilidad al agua por parte de estos materiales pizarrosos, puede hacerse patente a través de diversas manifestaciones (desmoronamiento, hinchamiento, disminución de la resistencia a la compresión, etc.), que influyen de una manera decisiva sobre la alterabilidad, a corto o largo plazo, de la roca $(1,2,3,4,5,6$ y 7 , entre otros).

En este sentido, Muñoz de la Nava (8) cita la necesaria determinación del peso específico y la absorción de agua, dentro de los diversos ensayos destinados a definir la calidad mecánica y su relación con la alterabilidad en rocas ornamentales, propiedades éstas que pueden hacerse extensivas a las pizarras de techar (9).

No obstante pocos autores $(10,11,12,13$ y 14 , entre otros), efectúan interpretaciones petrofisicas de la respuesta de los materiales pizarrosos frente a los fenómenos hídricos, siendo necesaria su realización si se desea predecir el comportamiento de la roca frente al agua.

La metodología de estudio aquí propuesta puede ser aplicada a cualquier material pizarroso que, por sus características de puesta en obra, esté en contacto con el agua.

Primeramente se ha efectuao un estudio detallado de las caracteristicas microscópicas (mineralogía, textura, estructura y microfisuración), aplicando diversas técnicas. Posteriormente, se han calculado algunas propiedades físicas relacionadas con el movimiento de fluidos a través de los materiales rocosos, interpretándolas en función de las características intrínsecas del material. Finalmente, se han realizado varios ensayos de alterabilidad, interpretándolos petrofísicamente.

Los estudios se realizaron sobre muestras de "roca matriz", (según terminología de la Sociedad Internacional de Mecánica de las Rocas).

\section{MATERIALES ESTUDIADOS}

Las explotaciones de las filitas de Rande, están 
situadas en Rande (La Coruña), siendo su principal uso como pizarra de techar y ornamental. La extracción de los bloques se realizó en una cantera en explotación con explosión controlada, para posteriormente utilizar sierras de hilo helicoidal y cuñas.

Las pizarras de Luarca (Asturias), presentan una gran uniformidad de facies en toda la región; las muestras estudiadas se han recogido en el borde Este del Cabo Peñas; su utilización principal es como pizarra de techar y ornamental; la extracción se realizó mediante cuñas.

Los estudios petrográficos (macro y microscópicos) y análisis semicuantitativos por difracción de Rayos $X$ han servido de base para la clasificación y descripción de las rocas estudiadas. En la Tabla I se sintetizan las principales características petrográficas; una descripción más amplia puede verse en (15).

TABLA I

Características petrográficas

\begin{tabular}{|l|l|l|}
\cline { 2 - 3 } \multicolumn{1}{c|}{} & \multicolumn{1}{c|}{ RANDE } & \multicolumn{1}{c|}{ LUARCA } \\
\hline $\begin{array}{l}\text { Descripción } \\
\text { petrográfica }\end{array}$ & $\begin{array}{l}\text { Filita gris oscura, es- } \\
\text { quistosidad planar } \\
\text { muy regular, orienta- } \\
\text { ción preferente de } \\
\text { los granos minera- } \\
\text { les; muy poca micro- } \\
\text { fisuración. }\end{array}$ & $\begin{array}{l}\text { Pizarra negra, den- } \\
\text { sa, esquistosidad } \\
\text { co microfisurada. } \\
\text { co pular; muy po- }\end{array}$ \\
\hline $\begin{array}{l}\text { Composi- } \\
\text { ción mine- } \\
\text { ralógica }\end{array}$ & $\begin{array}{l}\text { Cuarzo, clorita, illita, } \\
\text { moscovita, materia } \\
\text { orgánica, accesorios. }\end{array}$ & $\begin{array}{l}\text { Cuarzo, clorita, illita, } \\
\text { moscovita, sericita, } \\
\text { materia orgánica, ac- } \\
\text { cesorios. }\end{array}$ \\
\hline
\end{tabular}

TABLA II

Propiedades físicas de las rocas estudiadas: $\left(\rho_{g}\right)$ densidad de los granos minerales; $\left(\rho_{d}\right)$ densidad de la roca; $\left(n_{0}\right)$ porosidad abierta; $(n)$ porosidad total; (Sr) grado de relleno de los poros; ( $k 1$ ) permeabilidad al aire en sequedad;

(k2) permeabilidad al aire en condiciones ambientales

\begin{tabular}{|l|c|c|}
\cline { 2 - 3 } \multicolumn{1}{c|}{} & RANDE & LUARCA \\
\hline$\rho_{\mathrm{g}}\left(\mathrm{kg} / \mathrm{m}^{3}\right)$ & 2.780 & 2.810 \\
$\rho_{\mathrm{d}}\left(\mathrm{kg} / \mathrm{m}^{3}\right)$ & 2.750 & 2.740 \\
$\mathrm{n}_{\mathrm{o}}(\%)$ & 1,06 & 2,54 \\
$\mathrm{n}(\%)$ & 1,07 & 2,56 \\
$\mathrm{Sr}(\%)$ & 99,06 & 99,21 \\
$\mathrm{k} 1(\mu \mathrm{dcy})$ & 0,9 & 1,5 \\
$\mathrm{k} 2(\mu \mathrm{dcy})$ & 0,3 & 0,7 \\
\hline
\end{tabular}

Las propiedades físicas se han determinado siguiendo los métodos sugeridos por la ISRM (16); la permeabilidad al aire se ha medido mediante un permeámetro discontínuo de carga variable (17), realizándose las medidas perpendicularmente a la pizarrosidad, en sequedad total y condiciones ambientales $\left(20^{\circ} \mathrm{C}\right.$ y $75 \%$ de humedad).

Los resultados obtenidos aparecen en la Tabla II.

\section{COMPORTAMIENTO HIDRICO}

Para caracterizar la sensibilidad frente al agua de los materiales pizarrosos existen diversos ensayos (18); de entre ellos, se han realizado: contenido en agua de saturación, absorción de agua, desorción de agua, hinchamiento, desmoronamiento y ciclos de humedad/sequedad.

\section{Contenido en agua de saturación}

Para su determinación se han seguido el método sugerido por la ISRM (16) y (19); los valores se expresan en la Tabla III.

TABLA III

Contenido de agua en saturación (Ws); contenido de agua en absorción libre para una hora (Iv), a dos días $\left(W_{2}\right)$ y a ocho días $\left(W_{8}\right)$; contenido de agua en desorción (Wd)

\begin{tabular}{|l|l|l|}
\cline { 2 - 3 } \multicolumn{1}{c|}{} & RANDE & LUARCA \\
\hline Ws (\%) & 0,35 & 0,87 \\
Iv (\%) & 0,06 & 0,30 \\
W $_{2}(\%)$ & 0,16 & 0,37 \\
W $_{8}(\%)$ & 0,22 & 0,78 \\
Wd $(\%)$ & 0,03 & 0,21 \\
\hline
\end{tabular}

\section{Absorción libre de agua}

En este apartado se ha considerado: absorción libre de agua en una hora (índice de vacíos), en dos días y en ocho días; para su determinación se han seguido las recomendaciones de la ISRM (16) y (20). Los resultados pueden verse en la Tabla III.

Puede decirse, que tanto en saturación como en absorción libre de agua, ambos materiales presentan unos valores muy bajos, alcanzando un valor máximo las pizarras de Luarca, en condiciones de saturación del 0,87\%.

Asimismo, se ha constatado que a los ocho 
dias de absorción libre los valores determinados no alcanzan las condiciones de saturación (un $63 \%$ de saturación total en el caso de Rande y un $90 \%$ en el caso de las pizarras de Luarca).

La cinética de absorción libre de agua para tiempos largos (8 días), puede verse en las Figuras 1 y 2; en ellas se observa que la mayor captación de agua se realiza en las primeras horas de ensayo, alcanzando casi el $80 \%$ del valor total; esta rapidez de absorción viene condicionada preferentemente por el elevado grado de relleno de los poros $(\mathrm{Sr} \simeq 99$ \%) y el relativo gran tamaño de los mismos.

\section{Desorción de agua}

Posteriormente a la determinación del conte-

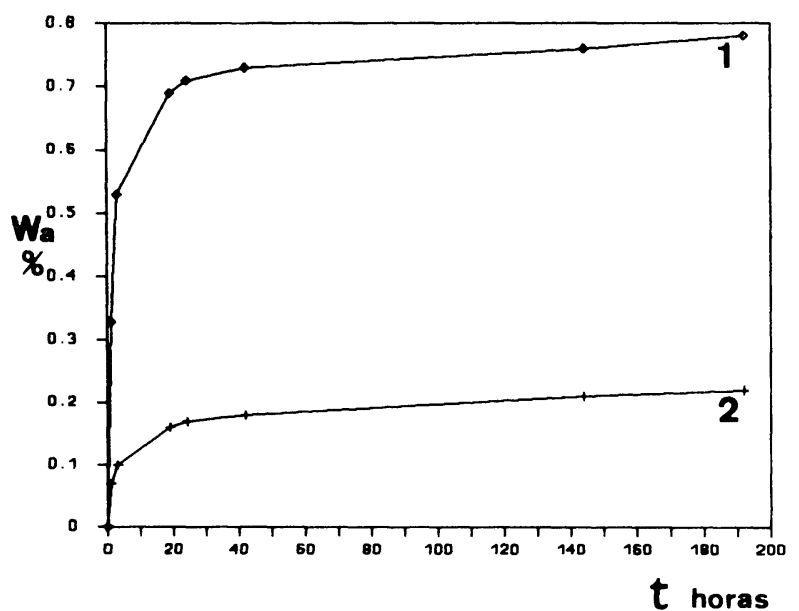

Fig. 1.-Variación del contenido de agua en absorción libre (Wa) frente al tiempo (t). (1) Pizarra de Luarca, (2) Filita de Rande.

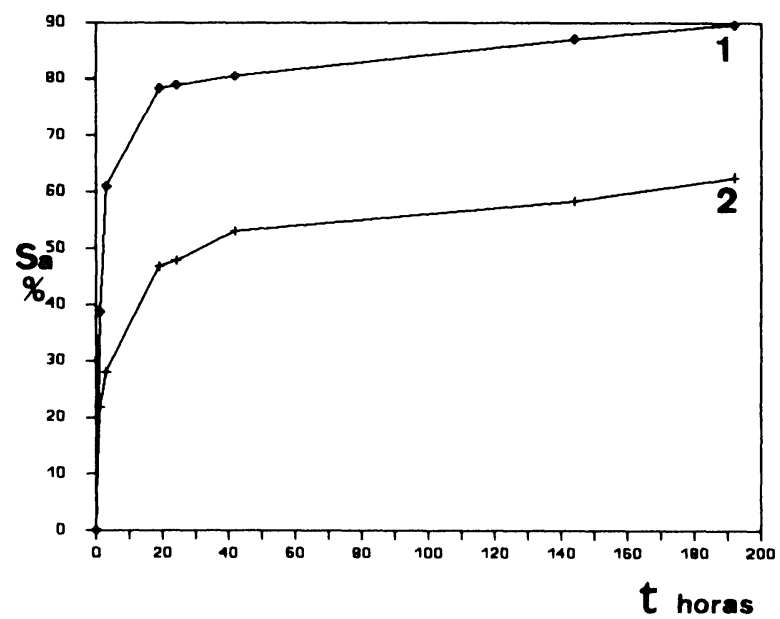

Fig. 2.-Variación del grado de saturación en absorción libre (Sa) frente al tiempo (t). (1) Pizarra de Luarca, (2) Fillita de Rande. nido de agua en saturación, se realizó el proceso inverso, en el cual las muestras se secaron al aire $\left(20^{\circ} \mathrm{C}\right.$ y $75 \%$ de humedad), durante 8 dias.

Los resultados para el contenido en agua final pueden verse en la Tabla III.

La cinética de la desorción para tiempos largos, puede verse en las Figs. 3 y 4.

La desorción se realiza con una cinética similar a la absorción libre, mostrando a las 20 horas de iniciado el ensayo, una pérdida de agua de aproximadamente un $80 \%$ del total.

Al final del ensayo, puede decirse que la filita de Rande, pierde un $92 \%$ del agua absorbida en saturación, mientras que las pizarras de Luarca, pierden un $76 \%$ del agua total.

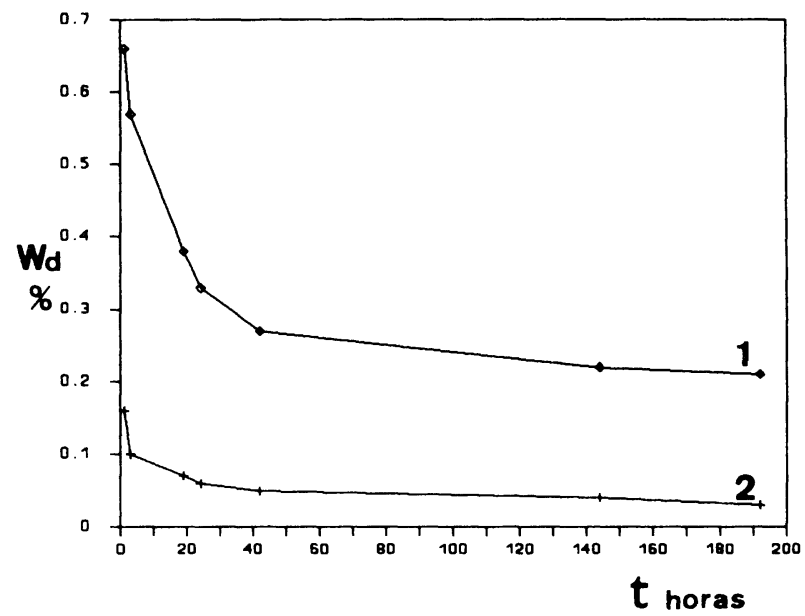

Fig. 3.- Variación del contenido de agua en desorción (Wd) frente al tiempo (t). (1) Pizarra de Luarca, (2) Filita de Rande.

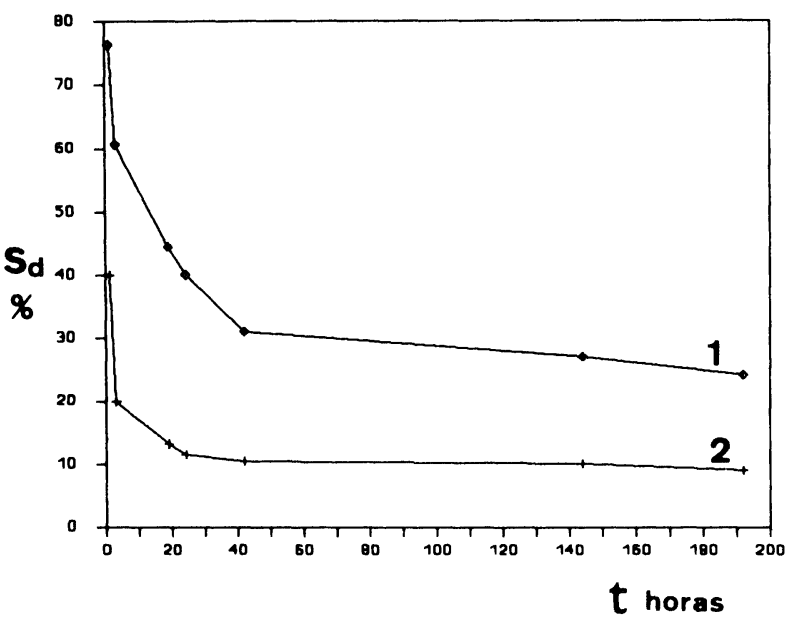

Fig. 4.-Variación del grado de saturación en desorción (Sd) frente al tiempo (t). (1) Pizarra de Luarca, (2) Filita de Rande. 


\section{Hinchamiento}

En la Tabla IV, aparecen los resultados de las medidas de hinchamiento libre (21), así como la anisotropía del mismo (A), según tres direcciones ortogonales $\left(\varepsilon_{\mathrm{s} 1}\right.$, perpendicular a la pizarrosidad y $\varepsilon_{\mathrm{s} 2}=\varepsilon_{\mathrm{s} 3}$, paralelos a la misma).

TABLA IV

Coeficientes de hinchamiento libre

$\left(\varepsilon_{s 1}, \varepsilon_{s 2}=\varepsilon_{s 3}\right)$ y coeficiente de anisotropia de hinchamiento $(A)$.

\begin{tabular}{|l|c|c|}
\cline { 2 - 3 } \multicolumn{1}{c|}{} & RANDE & LUARCA \\
\hline$\varepsilon_{\mathrm{s} 1} \times 10^{-3}$ & 0,18 & 1,00 \\
$\varepsilon_{\mathrm{s} 2}=\varepsilon_{\mathrm{s} 3} \times 10^{-3}$ & 0,00 & 0,37 \\
$\mathrm{~A}$ & $\infty$ & 1,70 \\
\hline
\end{tabular}

Los valores de hinchamiento son muy pequeños, los que está relacionado con el bajo contenido en minerales arcillosos relativamente expansivos ( $8 \%$ de illita en la filita de Rande y $12 \%$ de illita en las pizarras de Luarca). En este sentido, la mayor expansión mostrada por las pizarras de Luarca es debido, además del mayor porcentaje de illita, a la mayor abundancia de clorita, que favorece la mayor capacidad de cambio de cationes aumentando el hinchamiento, así como a una mayor porosidad y permeabilidad.

Por otra parte, ambas rocas muestran un comportamiento anisótropo que está favorecido por: variación de tamaño de grano, alineación de poros y microfisuras, bandeado composicional y orientación de minerales filosilicatados.

\section{Desmoronamiento}

Con el fin de evaluar la susceptibilidad al desmoronamiento, se realizó el ensayo de Lutton (22), que establece una serie de índices que corresponden a diferentes respuestas de los materiales pizarrosos por inmersión en agua.

Tanto las muestras de Rande, como las de Luarca, tienen un índice 6 (materiales que no sufren cambios aparentes).

\section{Ciclos de humedad-sequedad}

Los cambios repetidos en las condiciones de humedad, es uno de los factores determinantes de la alterabilidad de las pizarras.
Para la realización de los ensayos se ha seguido el método propuesto por Farjallat y de Oliveira (23); el tiempo de inmersión en agua y el de secado fue de 24 horas, realizándose éste a $20^{\circ} \mathrm{C}$ y $75 \%$ de humedad y siendo el número de ciclos de 20.

Con el fin de determinar el posible incremento de la microfisuración por los ciclos de humedad-sequedad, se realizó un estudio microscópico de la fracturación y desintegración, determinándose además el peso inicial y final de las muestras secas, así como el contenido en agua al finalizar la primera $\left(W_{0}\right)$ y la última absorción $\left(\mathrm{W}_{20}\right)$.

Siguiendo la clasificación de Deo et al. (24), ambas variedades se incluirian en el grupo 3 (materiales no afectados por el agua después de cinco ciclos). La pérdida de peso a lo largo de los ciclos fue prácticamente nula (filita de Rande, 0,003\% y pizarra de Luarca, 0,008\%).

Los resultados para la variación en el contenido en agua pre y post-ciclos, se muestran en la Tabla V. Puede verse que en el caso de la filita de Rande al cabo de 20 ciclos, el contenido en agua aumenta un $250 \%$ y en las pizarras de Luarca aproximadamente un $320 \%$. Estos resultados se corresponden con los estudios microscópicos post-ciclos realizados al SEM, en los que se ha visto la creación de algunas microfisuras de longitud y anchura variable, en general paralelas a la pizarrosidad.

TABLA V

Contenido en agua de absorción, antes $\left(W_{0}\right)$ y después de los ciclos de humedad-sequedad $\left(W_{20}\right)$

\begin{tabular}{|c|c|c|}
\cline { 2 - 3 } \multicolumn{1}{c|}{} & RANDE & LUARCA \\
\hline $\mathrm{W}_{0}$ (inicial) \% & 0,12 & 0,34 \\
$\mathrm{~W}_{20}$ (final) \% & 0,30 & 1,08 \\
\hline
\end{tabular}

\section{CONCLUSIONES}

En base a los estudios realizados puede decirse que ambas variedades presentan una buena respuesta frente al agua, interprentando dicho comportamiento como debido preferentemente a la casi ausencia de minerales arcillosos expansivos, así como su baja porosidad abierta y permeabilidad al aire.

Se ha podido comprobar que los cambios en el contenido en agua de estas rocas influyen sobre su alterabilidad. Así, los sucesivos ciclos de inmersión en agua-sequedad ocasionan 
expansiones y contracciones que provocan el incremento de la microfisuración. Dicho incremento microfisural está relacionado con las propiedades físicas de estos materiales; en este caso, las pizarras de Luarca, aún presentando una muy baja degradación mecánica, es cualitativa y cuantitativamente más alterable que la filita de Rande, cuyo comportamiento frente al agua es mucho mejor.

Así pues, ambos materiales pueden ser definidos desde el punto de vista geomecánico, como rocas muy poco afectadas por el agua, de escaso hinchamiento y gran durabilidad; según la clasificación propuesta por Duncan (25) para materiales pizarrosos, ambos materiales se encuadran dentro de los considerados como "endurecidos" ("indurated").

Se ha podido constatar una estrecha relación entre las características petrográficas (mineralogía, textura y estructura) y las propiedades físicas estudiadas.

Para evaluar la durabilidad de los materiales pizarrosos frente al agua, parece recomendable conocer diversas características petrográficas (naturaleza y proporción de la fracción arcillosa y anisotropía textural), propiedades fisicas (permeabilidad e hinchamiento) así como el comportamiento de dichos materiales al verse sometidos a ciclos de humedad-sequedad.

\section{BIBLIOGRAFIA}

(1) EECKOUT, E. M. (1976): The mechanisms of strength reduction due to moisture in coal mine shale. Int. J. Rock Mech. Min. Sci. \& Geomech. Abstr., 13, pp. 61-67.

(2) OLIVER, H. J. (1979): Some engineering aspects of tunnel construction in Karoo strata with special reference to the Orange-Fish tunnel. Ph. D. Thesis Univ. of the Orange Free State Bloemfontein, U.S.A., 383 pp.

(3) HARPER, T. R.; APPEL, G.; PENDLETON, M. W.; SZYMANSKI, J. S. y TAYLOR, R. K. (1979): Swelling strain development in sedimentary rock in Northern New York. Int. J. Rock Mech. Min. Sci. \& Geomech. Abstr., 16, pp. 271-292.

(4) LOCAT, J.; LEFEBVRE, G. y BALLIUY, G. (1984): Mineralogy, chemistry and physical properties interrrelationships of some sensitive clays from Eastern Canada. Canadian Geotechnical Journal, 21, 3, pp. 530-540.

(5) ATABEK, R.; JOIDA, M. y ANDRE-JEHAN, R. (1985): Programs and means developed by C.E.A. for clay characterization. Engineering Geology, 21, pp. 209-213.

(6) CRIPPS, J. C. y TAYLOR, R. K. (1986): Engineering characteristics of British over-consolidated clays and mudrocks, I. Tertiary deposits. Eng. Geology, 22, pp. 349-376.

(7) CRIPPS, J. C. y TAYLOR, R. K. (1987): Engineering characteristics of British over-consolidated clays and mudrocks, II. Mesozoic deposits. Eng. Geology, 23, pp. 213-253.

(8) MUÑOZ DE LA NAVA, P.; ROMERO ESCUDERO, J. A.; RODRÍGUEZ SUÁREZ, I.; GARCÍA ROMERO, E.; ROSA CRESPO, A.; CARRIÓN MOLES, F. y GARBAYO MARTÍNEZ, M. P. (1989): Metodologia de investigación de piedras naturales: granitos. Roc Maquina, 4..$^{\circ}$ Trimestre, pp. 14-44.

(9) UNE 22-191-85 (1985): Pizarras ornamentales. Placas y Losas. Absorción y peso específico aparente. Instituto Español de Normalización (CSIC).

(10) OKAMOTO, R.; SUGAHARA, H. y HIRANO, I. (1981): Slaking and swelling properties of mudstone. K. AKAI, M. HAYASHI \& Y. NISHIMATSU (Eds.). Proc. of the Int. Symp. on Weak Rock (Tokyo), A. A. BALKEMA (Rotterdam), pp. 213-218.

(11) SASAKI, T.; KINOSHITA, S. y ISHIJIMA. Y. (1981): A study on water-sensivity of argillaceous rock. K. AKAI, M. HAYASHI \& Y. NISHIMATSU (Eds.). Proc. of the Int. Symp. on Weak Rock (Tokyo), A.A. BALKEMA (Rotterdam), pp. 149-154.

(12) HUANG, S. L.; AUGHENBAUGH, N. B. y ROCKAWAY, J. D. (1986): Swelling pressure studies of shales. Int. J. Rock Mech. Min. Sci. \& Geomech. Abstr., 23, pp. 371-377.

(13) RUIZ DE ARGANDOÑA, V. G.; MONTOTO, M.; RODRÍGUEZ-REY, A. y CALLEJA, L. (1988): Caracterización petrofísica de rocas arcillosas expansivas. Proc. of the Int. Cong. on "Tunnels and Water" (Madrid). J. M. SERRANO (Eds.). A. A. BALKEMA (Rotterdam), pp. 299-306.

(14) SWAN, G.; COOK, J. y BRUCE, S. (1989): Strain rate effects in Kimmeridge Bay shale. Int. J. Rock Mechanics Min. Sci \& Geomech. Abstr. Vol. 26, pp. 135-149.

(15) RUIZ DE ARGANDOÑA, V. G. (1980): Estudio sobre las características físicas y de alterabilidad frente al agua de algunos materiales pizarrosos. Tesis de Licenciatura, Facultad de Geologia, Univ. de Oviedo. 135 pp.

(16) I.S.R.M. (1981): Rock characterization testing and monitoring. E. T. Brown (Eds.). En: I.S.R.M. Suggested methods, $211 \mathrm{pp}$. 
(17) THENOZ, B. (1956): Mesure de la permeabilité et de la porosité des roches trés compactes. C.R.Ac.Sc.Fr., 243, pp. 289-291.

(18) DAPENA, J. E. y URIEL, S. (1980): Determinación de la meteorabilidad de las rocas. Bol. Inf. Lab. Carret. y Geotecnia, 142, pp. 3-34.

(19) BELIKOV, B. P.; ZALESKII, B. V.; ROZANOV, Y. A.; SANINA, E. A. y TIMCHENKO, I. P. (1967): Methods of studing the physicomechanical properties of rocks. In: Physical and mechanical properties of rocks. Israel Program for Scientific Traslation. Jerusalem. $152 \mathrm{pp}$.

(20) C.N.R.-I.C.R. (Centro di studio cause di diperimento e metodi de conservazione delle opera d'Arte - Instituto Centrale del Restauro) (1981). Assorbimento d'acqua per immersione totale. Capacitá di imbibizioni. Doc. Normal: 7/81, Roma, 5 pp.

(21) I.S.R.M. (1989): Suggested methods for laboratory testing of argillaceous swelling rocks. Int. J. Rock Mech. Min. Sci. \& Geomech. Abstr., 26, pp. 415-426.

(22) LUTTON, R. J. (1977): Slaking index for desing. In: Desig and construction of compacted shale embankment, 3. U.S. Army Eng. Water Ways. Exp. Station, Federal Highway Administration.

(23) FARJALLAT, J. y DE OLIVEIRA, N. (1972): Experimental studies on the weatherability of the Capivara dam basalts, Rios Paranapanema, Brazil. Bull. Int. Assoc. Engng. Geol., 6, pp. 83-96.

(24) DEO, P.: WOOD, L. E. y LOWELL, Jr. C.W. (1974): Use of shale in embankments. National Research Board, Special Report, 148, pp. 87-96.

(25) DUNCAN, N. (1969): Engineering geology and rock mechanics. Leonards Hill (Eds.). Vol. 1-2. Londres, 522 pp.

\section{publicación del ICCET/CSIC}

\section{ACUEDUCTOS ROMANOS EN ESPAÑA \\ Carlos Fernández Casado}

Prof. Dr. Ing. de Caminos, Canales y Puertos

Esta publicación se compone de una serie de articulos, publicados en la Revista "Informes de la Construcción", en los cuales se hace un análisis de los acueductos romanos que existen en España y el balance de las condiciones de conservación en que se encuentra cada uno de ellos, incluyendo referencias históricas y literarias. Se ha ilustrado con la reproducción de la valiosa documentación gráfica que posee el prestigioso autor.

Un volumen encuadernado en couché, a dos colores, de $21 \times 27$ centimetros, compuesto de 238 páginas, numerosos grabados, dibujos, fotos en blanco y negro y figuras de linea.

Precio: España, 1.500 ptas., 21 \$ USA.

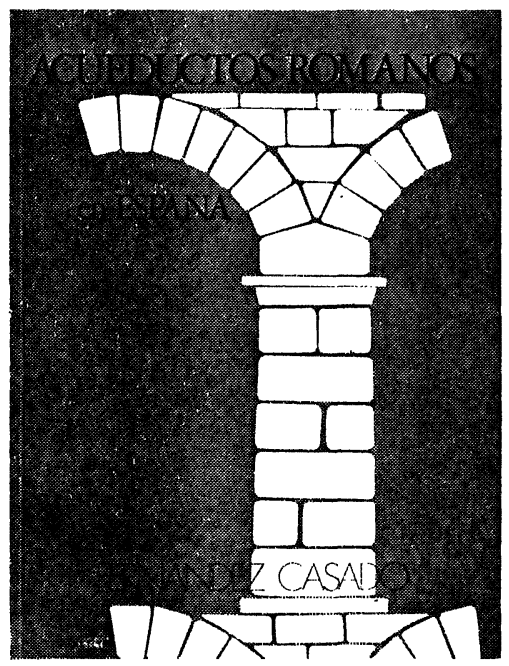

